\title{
DESENVOLVIMENTO INICIAL DE PLANTAS DE NONI CULTIVADAS EM DIFERENTES SUBSTRATOS
}

\author{
Marcela Sant'Anna Cordeiro da Silva ${ }^{1}$, Aparecida Conceição Boliani ${ }^{2}$, Antonio Flávio \\ Arruda Ferreira ${ }^{3}$, Laís Naiara Honorato Monteiro ${ }^{4}$, Regina Maria Monteiro de Castilho ${ }^{2}$
}

\footnotetext{
${ }^{1}$ Mestranda em Agronomia: Horticultura - Universidade Estadual Paulista "Júlio de Mesquita Filho" - FCA, Botucatu/SP.

${ }^{2}$ Professora Dra. do Departamento de Fitotecnia, Tecnologia de Alimentos e Sócio Economia - Universidade Estadual Paulista "Júlio de Mesquita Filho" - FEIS, Ilha Solteira/SP.

${ }^{3}$ Doutorando em Fitotecnia - Universidade de São Paulo - ESALQ, Piracicaba/SP.

${ }^{4}$ Mestranda em Agronomia: Sistemas de Produção - Universidade Estadual Paulista "Júlio de Mesquita Filho" - FEIS, Ilha Solteira/SP.
}

RESUMO: O noni (Morinda citrifolia L.) é uma espécie de grande importância medicinal que possui ampla distribuição pelo mundo. Suas propriedades fitoterápicas englobam dentre várias utilidades, o tratamento de diabetes, hipertensão, alergias, depressão e câncer. Apesar do sucesso da planta, ainda são escassas as informações a respeito de suas características agronômicas no Brasil e com isso, a produção de mudas de qualidade, torna-se um desafio. Neste sentido, objetivou-se com o presente trabalho, avaliar os efeitos de composições de substratos na produção de mudas de noni. O experimento foi desenvolvido em casa de vegetação (Pad\&Fan) da UNESP, Câmpus de Ilha Solteira - SP, no período de 16 de setembro a 16 de dezembro de 2014, sendo o delineamento experimental inteiramente casualizado, composto por 4 tratamentos com 6 repetições de 4 plantas. Os substratos utilizados foram: S1 - solo + areia (1:1); S2 - solo + areia + matéria orgânica (1:1:1); S3 - solo + matéria orgânica (1:1); S4 - solo + areia (1:1) + Osmocote ${ }^{\circledR} 15-09-12\left(4,0 \mathrm{~g} \mathrm{~L}^{-1}\right)$. Avaliou-se: comprimento de parte aérea (CPA), diâmetro de caule (DC), relação comprimento de parte aérea/diâmetro de caule (RCPA/DC) e índice SPAD. Os resultados foram submetidos à análise de variância e regressão utilizando o programa SISVAR 5.3 para análise dos dados e as médias comparadas pelo teste de Tukey a $5 \%$ de probabilidade. Concluiu-se que o melhor substrato para a formação de mudas de noni foi substrato S4, pois proporcionou maior CPA, DC, índice SPAD e valores ideais para RCPA/DC.

Palavras-chave: Morinda citrifolia L. Qualidade de muda. Fertilizante de liberação lenta.

\section{INITIAL DEVELOPMENT OF NONI CULTIVATED PLANTS IN DIFFERENT SUBSTRATES}

\begin{abstract}
Noni (Morinda citrifolia $\mathrm{L}$.) is a species of great medical importance, which a wide distribution throughout the world. Its herbal properties include among several uses, the treatment of diabetes, hypertension, allergies, depression and câncer. Despite the success of the plant, there is still little information about their agronomic characteristics in Brazil, and with it, the production of quality seedlings, it becomes a challenge. In this sense, the objective of this study was to evaluate the effects of compositions of substrates in the production of
\end{abstract}

Cultura Agronômica, Ilha Solteira, v.25, n.3, p.241-252, 2016 
noni seedlings. The experimental was conducted in greenhouse (Pad\&Fan) of UNESP, Ilha Solteira - SP, in the period of september 16 to december 16, 2014, and the design was completely randomized, with 4 treatments with 6 replicates of 4 plants. The substrates used were: S1 - soil + sand (1:1); S2 - soil + sand + organic matter (1:1:1); S3 - soil + organic matter (1:1); S4 - soil + sand (1:1) + Osmocote ${ }^{\circledR} 15-09-12\left(4,0 \mathrm{~g} \mathrm{~L}^{-1}\right)$. Were evaluated: shoot length (CPA), stem diameter (DC), shoot length/diameter ratio (RCPA/DC) and SPAD index. The results were submitted to variance analysis and regression using the SISVAR 5.3 program for data analysis and avarages were compared by means of Tukey test at $5 \%$ probability. It was concluded that the best substrate for the formation of noni seedlings was S4 substrate, because it provided greater CPA, DC, SPAD index and optimal values for RCPA/DC.

Key words: Morinda citrifolia L.. Seedlings quality. Slow release fertilizer.

\section{INTRODUÇÃO}

O noni, cientificamente conhecido por Morinda citrifolia L., é uma espécie frutífera pertencente à família Rubiaceae, originária do sudeste asiático, que vem se destacando pelo mundo não somente por ser importante fonte nutricional, mas principalmente por possuir inúmeras propriedades fitoterápicas, dentre as quais pode-se citar seu alto poder antioxidante, antibacteriano, antiviral, antifúngico, antitumoral, anti-helmíntico, analgésico, antiinflamatório e imunoestimulante (BASAR et al., 2010; NASCIMENTO, 2012).

Sua introdução no Brasil deu-se há poucos anos e em função da demanda pelos produtos oriundos da planta, a produção do noni em pomares domésticos e comerciais já tornou-se realidade no país. Apesar disso, informações a respeito de sua propagação e consequente produção de mudas são escassas (SOUSA et al., 2009; SILVA et al., 2012).

Nesse contexto, a produção de mudas de noni estabelece uma etapa crucial do processo produtivo, uma vez que influencia o desempenho das plantas além de seus produtos finais. $\mathrm{O}$ tipo de substrato utilizado é um fator determinante que afeta diretamente o desenvolvimento das plantas, posto que deve reunir atributos que supram a necessidade da planta (BRASIL et al., 1999; BARBOSA et al., 2003; CUNHA et al., 2006).

Em geral é difícil encontrar um material que isoladamente atenda as exigências nutricionais da espécie, posto que trata-se de um aspecto essencial para as plantas não terem crescimento prejudicado. Em geral, pode-se dizer que é preferível a mistura de dois ou mais materiais melhoradores das características físicas e/ou químicas, para que se obtenha um substrato adequado e de boa qualidade (BACKES, 1989; KÄMPF, 1992; GONÇALVES et al., 2000; SANTOS et al., 2000).

Diante da ausência de informações sobre o cultivo da espécie, objetivou-se com o presente trabalho avaliar os efeitos de composições de substratos na produção de mudas de noni. 


\section{MATERIAL E MÉTODOS}

O experimento foi realizado no período de 16 de setembro a 16 de dezembro de 2013 em casa de vegetação (Pad\&Fan), com temperatura de $28,0 \pm 2,0{ }^{\circ} \mathrm{C}$ e umidade relativa do ar de 75,0 \pm 5,0 \%, pertencente a Faculdade de Engenharia da Universidade Estadual Paulista “Júlio de Mesquita Filho" (UNESP), Câmpus de Ilha Solteira - SP, latitude 20²5'28” S, longitude $51^{\circ} 21^{\prime} 15^{\prime \prime} \mathrm{W}$ e altitude de $354 \mathrm{~m}$.

Noventa dias após a semeadura, mudas de noni foram transplantadas para sacos plásticos com capacidade de 1 litro, preenchidos com substratos a base de solo, areia e matéria orgânica.

Os substratos utilizados foram: S1 - Solo + Areia (1:1, v:v); S2 - Solo + Areia + Matéria Orgânica (1:1:1, v:v:v); S3 - Solo + Matéria Orgânica (1:1, v:v); S4 - Solo + Areia (1:1, v:v) + Osmocote ${ }^{\circledR} 15-09-12$ (4,0 $\mathrm{g} \mathrm{L}^{-1}$ substrato).

O solo utilizado na composição dos substratos caracteriza-se por Latossolo VermelhoEscuro Distrófico (EMBRAPA, 2013), coletado em profundidade de 0,0 - 0,20 m na Fazenda de Ensino, Pesquisa e Extensão, da Faculdade de Engenharia - UNESP - Câmpus de Ilha Solteira. A areia utilizada foi do tipo média e lavada e a matéria orgânica utilizada foi produzida através da decomposição durante 90 dias da mistura de esterco bovino curtido, folhas de fícus (Ficus elástica Roxb. ex Hornem.) e aparos de grama batatais (Paspalum notatum Flügge.), na proporção 2:1:1 respectivamente. Previamente à montagem do experimento, o solo, areia e matéria orgânica foram passados em peneira de malha $4 \mathrm{~mm}$.

A Tabela 1 mostra a composição em porcentagem dos nutrientes contidos nos grânulos revestidos por resina biodegradável (Osmocote ${ }^{\circledR}$ ) utilizados na condução do experimento.

Tabela 1. Composição em porcentagem dos nutrientes contidos nos grânulos de Osmocote ${ }^{\circledR}$.

\begin{tabular}{ccccccccccc}
\hline $\mathrm{N}$ & $\mathrm{P}_{2} \mathrm{O}_{5}$ & $\mathrm{~K}_{2} \mathrm{O}$ & $\mathrm{Mg}$ & $\mathrm{S}$ & $\mathrm{Fe}$ & $\mathrm{B}$ & $\mathrm{Cu}$ & $\mathrm{Mn}$ & $\mathrm{Mo}$ & $\mathrm{Zn}$ \\
\hline \multicolumn{7}{c}{} & & \multicolumn{7}{c}{$(\%)$} \\
\hline 15,0 & 9,0 & 12,0 & 1,3 & 5,9 & 0,46 & 0,02 & 0,05 & 0,06 & 0,02 & 0,05 \\
\hline
\end{tabular}

Fonte: Empório das sementes (2014)

O sistema de irrigação adotado foi do tipo micro aspersão, utilizando-se de três irrigações diárias (6:00, 12:00 e 18:00 horas), com duração de cinco minutos cada e vazão de $1800 \mathrm{~L} \mathrm{~min}^{-1}$. Realizou-se uma aplicação do inseticida de contato e ingestão, princípio ativo deltametrina e grupo piretróide, Decis Ultra $100 \mathrm{EC}$, na dosagem de $10 \mathrm{~mL} 20 \mathrm{~L}^{-1}$ de água para controle de pulgão.

O delineamento estatístico experimental utilizado foi o inteiramente casualizado, composto por 4 tratamentos com 6 repetições, sendo que cada unidade experimental foi composta por 4 plantas.

Foram feitas avaliações quinzenais das seguintes variáveis: comprimento de parte aérea (CPA) $(\mathrm{cm})$, medida entre a região do colo e o meristema apical, utilizando-se de régua graduada; diâmetro de caule (DC) (mm), medido na altura do colo das plantas, com o auxílio de um paquímetro; relação comprimento de parte aérea/diâmetro de caule (RCPA/DC)

Cultura Agronômica, Ilha Solteira, v.25, n.3, p.241-252, 2016 
(cm.mm), relação entre o valor de comprimento de parte aérea e valor de diâmetro de caule e soil plant analysis development (índice SPAD), com o auxílio de um clorofilômetro (Minolta SPAD 502), as medições foram feitas nas folhas mais expandidas localizadas região superior da planta, sendo uma folha por planta.

Os resultados foram submetidos à análise de variância e regressão utilizando o programa SISVAR 5.3 (FERREIRA, 2011) para análise dos dados e as médias comparadas pelo teste de Tukey a $5 \%$ de probabilidade.

\section{RESULTADOS E DISCUSSÃO}

As variáveis comprimento da parte área (CPA) e diâmetro do caule (DC) ajustaram-se às equações do tipo linear, enquanto que a relação comprimento de parte aérea/diâmetro de caule (RCPA/DC) ajustou-se ao modelo quadrático (Figura 1). O substrato S4 destacou-se em todas as respectivas variáveis, enquanto que o substrato $\mathrm{S} 1$ demonstrou contribuir pouco para o desenvolvimento das mudas de noni.
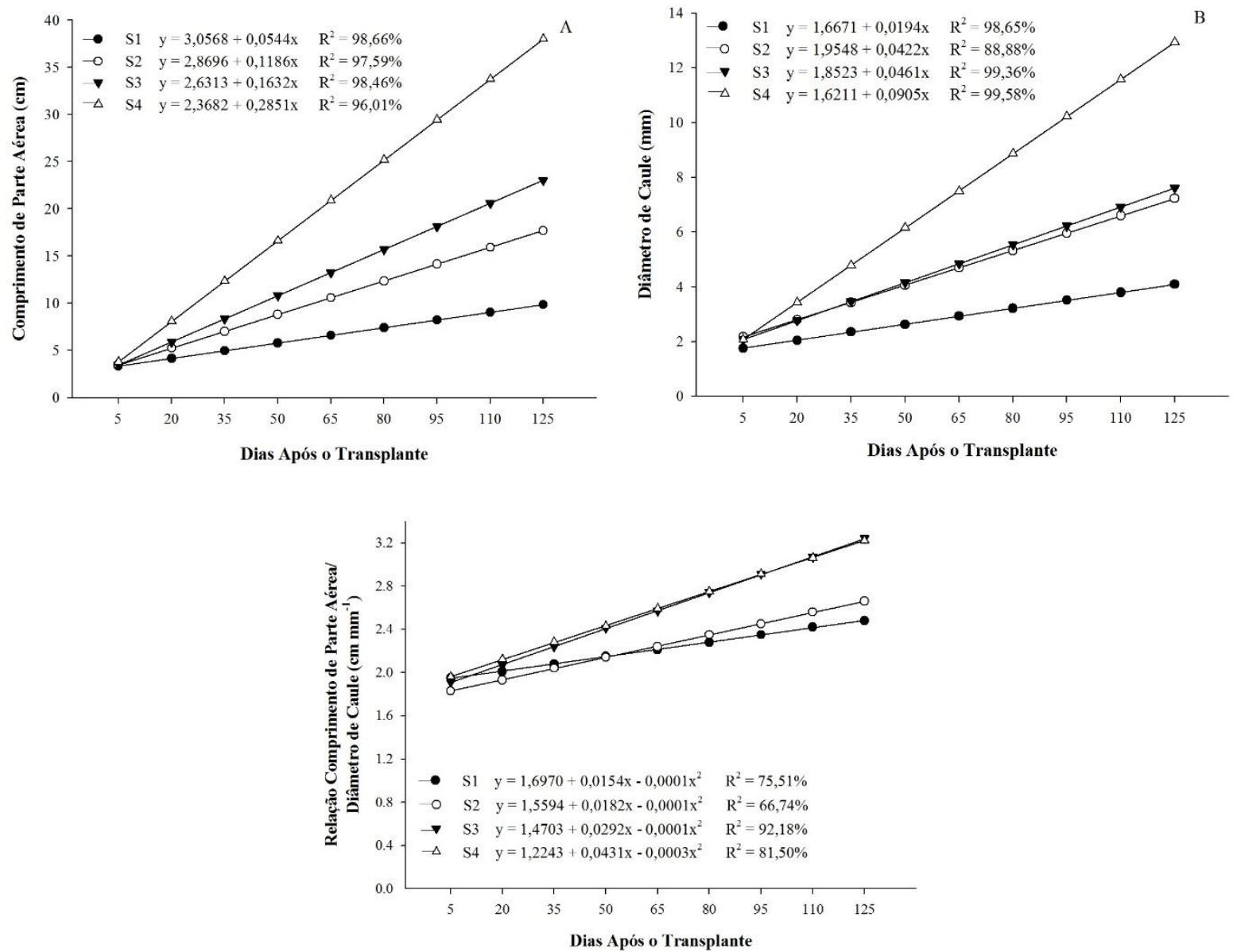

Figura 1. Comprimento de parte aérea, diâmetro de caule e relação comprimento de parte aérea/diâmetro de caule de plântulas de noni desenvolvidas em composições de substratos. S1: Solo + Areia (1:1); S2: Solo + Areia + Matéria Orgânica (1:1:1); S3: Solo + Matéria orgânica (1:1); e S4: Solo + Areia (1:1) + Osmocote ${ }^{\circledR} 15-09-12$ (4,0 g L ${ }^{-1}$ substrato).

Cultura Agronômica, Ilha Solteira, v.25, n.3, p.241-252, 2016 
Na Tabela 2 estão descritas as médias de comprimento de parte aérea, diâmetro de caule e relação comprimento de parte aérea/diâmetro de caule de plântulas de noni obtidas na última avaliação, aos 125 dias após o transplante, onde é possível melhor observação dos resultados obtidos.

Tabela 2. Comprimento de parte aérea, diâmetro de caule e relação comprimento de parte aérea/diâmetro de caule de plântulas de noni aos 125 dias após o transplante. Ilha Solteira SP, 2015.

\begin{tabular}{cccc}
\hline \multirow{2}{*}{ Substratos } & CPA & DC & RCPA/DC \\
\cline { 2 - 4 } & $(\mathrm{cm})$ & $(\mathrm{mm})$ & $\left(\mathrm{cm} \mathrm{mm}^{-1}\right)$ \\
\hline S1 & $9,98 \mathrm{~d}$ & $4,12 \mathrm{c}$ & $2,43 \mathrm{~b}$ \\
S2 & $17,84 \mathrm{c}$ & $6,81 \mathrm{~b}$ & $2,62 \mathrm{ab}$ \\
S3 & $22,44 \mathrm{~b}$ & $7,48 \mathrm{~b}$ & $3,03 \mathrm{a}$ \\
S4 & $35,18 \mathrm{a}$ & $12,62 \mathrm{a}$ & $2,80 \mathrm{ab}$ \\
\hline C.V. $(\%)$ & 11,30 & 5,56 & 11,55 \\
\hline DMS & 3,90 & 0,70 & 0,51
\end{tabular}

Médias seguidas pela mesma letra, na coluna, não diferem entre si, pelo teste Tukey a $5 \%$ de probabilidade de erro. C.V. = Coeficiente de Variação. DAT: Dias Após o Transplante. S1: Solo + Areia (1:1); S2: Solo + Areia + Matéria Orgânica (1:1:1); S3: Solo + Matéria orgânica (1:1); e S4: Solo + Areia (1:1) + Osmocote ${ }^{\circledR}$ 15-09-12 $\left(4,0 \mathrm{~g} \mathrm{~L}^{-1}\right.$ substrato).

Em relação a comprimento de parte aérea, observa-se que houve maior crescimento em altura das mudas no substrato $\mathrm{S} 4$, proporcionando altura de $35,18 \mathrm{~cm}$ ao final do experimento, seguido dos substratos S3, S2 e S1 (22,44; 17,84 e 9,98 cm respectivamente) (Tabela 2). A adição de matéria orgânica na composição do substrato S2 favoreceu o valor de CPA das mudas, quando contrastado com o substrato $\mathrm{S} 1$, que não possui este componente, uma vez que, segundo Negreiros et al. (2004) a associação de materiais orgânicos, melhora a textura do substrato e assim, propicia boas condições físicas, além de fornecer nutrientes necessários ao desenvolvimento das raízes e da muda.

A diferença de 25,20 cm no CPA das mudas entre os substratos S1 e S4, indica que a adição do fertilizante de liberação lenta Osmocote ${ }^{\circledR}$, formulação 15-09-12 na composição do substrato, contribuiu de forma eficiente para o melhor desenvolvimento das mudas (Figura 2). Este resultado pode ter decorrido em função do fertilizante Osmocote ${ }^{\circledR}$ liberar gradualmente os nutrientes, permanecendo disponível às plantas durante a maior parte do período de desenvolvimento das mudas, fazendo com que a nutrição destas seja prolongada, evitando a deficiência nutricional. 

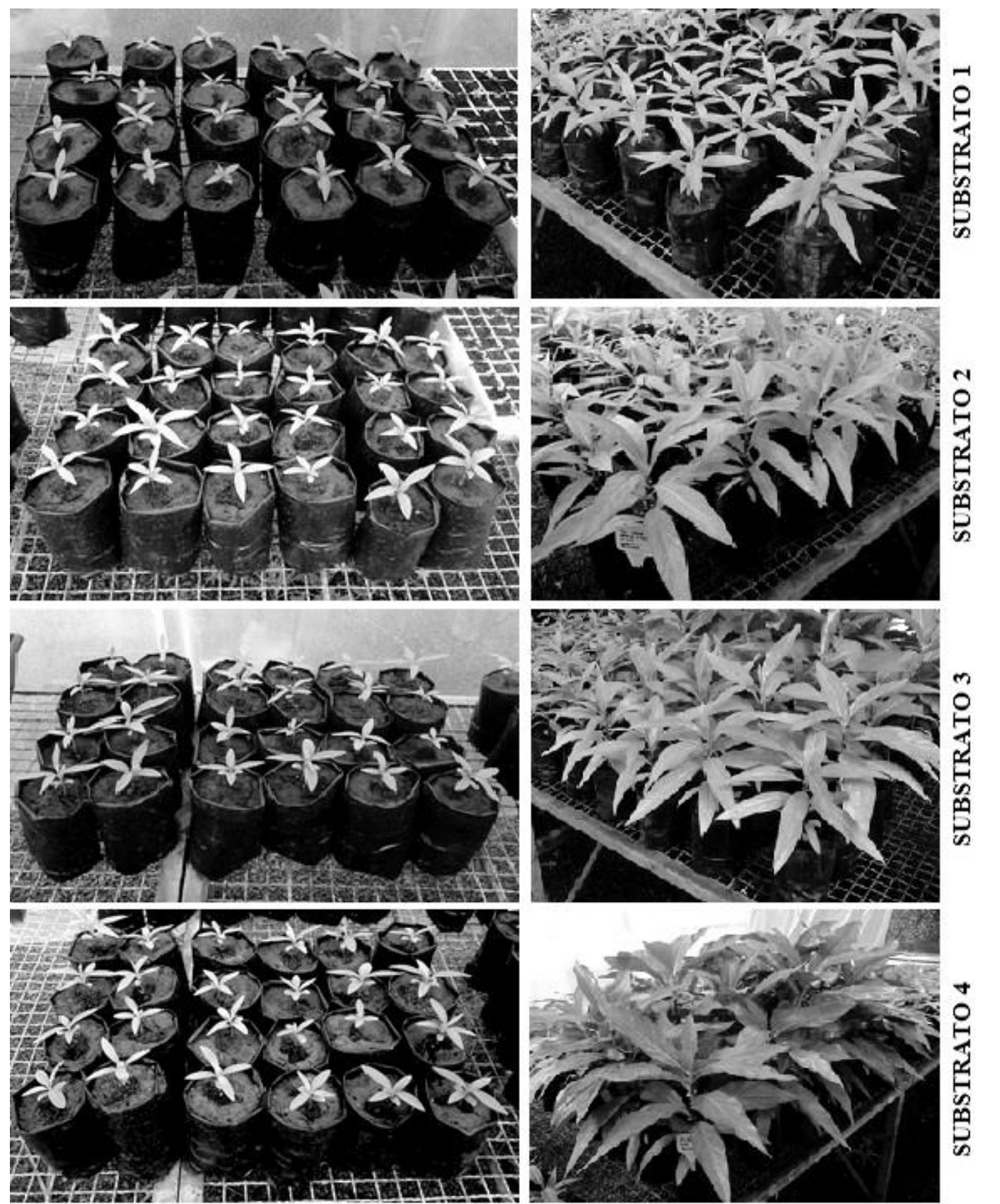

瓷

Figura 2. Plantas de noni desenvolvidas em composições variadas de substratos, logo após transplante (à esquerda) e ao final do experimento, com 125 dias após o transplante (à direita). Substrato 1: Solo + Areia (1:1); Substrato 2: Solo + Areia + Matéria Orgânica (1:1:1); Substrato 3: Solo + Matéria orgânica (1:1); e Substrato 4: Solo + Areia (1:1) + Osmocote ${ }^{\circledR}$ 15-09-12 (4,0 $\mathrm{g} \mathrm{L}^{-1}$ substrato). Ilha Solteira - SP, 2014.

Trabalhando com mudas de maracujazeiro (Passiflora edulis - Passifloraceae), Mendonça et al. (2004) constataram que mudas produzidas em substrato com a presença de Osmocote $^{\circledR}$ (15-10-10) em sua composição, obtiveram CPA significativamente superior, quando comparado às mudas produzidas sem a presença do fertilizante, o que corrobora com o presente estudo. Além disso, em trabalho realizado com a cultura do mamoeiro (Carica papaya - Caricaceae), Yamanishi et al. (2004) observaram resultados favoráveis no CPA com aplicação de Osmocote ${ }^{\circledR}$ em diferentes substratos.

Cultura Agronômica, Ilha Solteira, v.25, n.3, p.241-252, 2016 
Resultados diferentes foram encontrados por Dinalli et al. (2012) que avaliando a utilização de fertilizantes de liberação lenta em mudas de Vigna radiata L. (Fabaceae), verificaram que não houve diferença significativa do substrato contendo Osmocote ${ }^{\circledR}$ em relação à testemunha para CPA. O mesmo ocorreu com trabalho desenvolvido por Oliveira (2006) com a espécie Dianthus caryophyllus (Cravo - Caryophyllaceae) onde o resultado mais satisfatório foi observado para a testemunha.

Os resultados favoráveis relacionados à mistura de material orgânico ao substrato (substratos S2 e S3), podem ter influência da capacidade de fornecimento de nutrientes desse composto, que também promove a melhoria das características químicas, físicas e biológicas, criando um ambiente adequado para o crescimento das raízes e da planta como um todo (CASAGRANDE JÚNIOR et al., 1996). Em estudo realizado por Moreira et al. (2013), ficou comprovado que o emprego de composto orgânico como fertilizante, também é um manejo viável nutricional e economicamente para a cultura do noni.

Para diâmetro de caule, verificou-se que o substrato S4 contribuiu para um melhor desenvolvimento das mudas durante todo o experimento, proporcionando média de 12,62 $\mathrm{mm}$ na última avaliação, ao passo que o substrato S1 mostrou-se como o menos favorável, proporcionando 4,12 $\mathrm{mm}$. Estes valores evidenciaram diferença de 8,5 $\mathrm{mm}$ para DC entre ambos os substratos (Tabela 2).

Estes resultados estão de acordo com o encontrado por Santana Filho et al. (2003) em estudo realizado com plantas de noni, onde ficou evidente a resposta positiva da adubação realizada durante o experimento, que promoveu um crescimento contínuo e significativo do DC das plantas, com média máxima de 32,75 mm após 360 DAT. Resultado semelhante relacionados à adubação com Osmocote (15-10-10) foi observado por Marcuzzo et al. (2005), onde trabalhando com Coffea arabica (Café - Rubiaceae) verificou que o uso do fertilizante nas doses entre 453,04 e 506,08 g proporcionou melhores valores de DC, além de mudas mais desenvolvidas, independente do substrato utilizado.

Em relação ao uso de material orgânico nos substratos, Howard (2007) afirma que o noni responde à sua adição como complemento do equilíbrio nutricional, por ser importante fonte de nutrientes. Já em relação ao uso de areia, sabe-se que esta contribui de maneira eficaz com a drenagem do substrato, porém, não contém nutrientes. Sendo assim, pode-se dizer que o fato de o substrato $\mathrm{S} 1$ ter proporcionado menores médias indica que somente a composição solo + areia não é suficiente para garantir um bom desenvolvimento das mudas durante um período maior de tempo, sem que seja realizada uma reposição nutricional e que adicionando matéria orgânica ao meio (substrato S2), os resultados apresentam melhora. De acordo com Moreira e Moreira (1996), mudas DC menores que $3 \mathrm{~mm}$, apresentam dificuldades de se manterem eretas após o plantio.

A relação comprimento da parte aérea/diâmetro de caule exprime o equilíbrio de desenvolvimento das mudas no viveiro, pois conjuga duas características em apenas um só índice, além de fornecer informações de quão delgada está a muda (CARNEIRO, 1995; GOMES; PAIVA, 2004).

Cultura Agronômica, Ilha Solteira, v.25, n.3, p.241-252, 2016 
De acordo com Carneiro (1995), altos valores de RCPA/DC consistem num aspecto positivo, pois as mudas devem apresentar diâmetros maiores para maior equilíbrio do desenvolvimento da parte aérea. Baseando-se no contexto, verifica-se que o substrato S3 apresentou o melhor valor $\left(3,03 \mathrm{~cm} \mathrm{~m}^{-1}\right)$ seguido do substrato S4, S2 e S1 (2,80; 2,62 e 2,43 $\mathrm{cm} \mathrm{mm}^{-1}$ respectivamente) (Tabela 2 ).

Segundo Schmidt-Vogt e Gürth (1969), para o maior crescimento em parte aérea, as mudas devem apresentar compatível desenvolvimento em diâmetro, o que não ocorreu com os diâmetros das plantas submetidas aos substratos S3 e S4.

A variável índice SPAD não se ajustou à regressão, sendo suas médias representadas na Tabela 3. Nota-se que durante a condução do experimento, o substrato S4 demonstrou ser superior, proporcionando diferenças significativas em relação aos demais substratos na última avaliação (52,54). O substrato $\mathrm{S} 1$, por outro lado, proporcionou os menores valores médios para esta variável, obtendo índice de 38,71 na última avaliação.

Tabela 3. Valores médios para índice SPAD de plântulas de noni desenvolvidas em composições de substratos. Ilha Solteira - SP, 2015.

\begin{tabular}{cccccccc}
\hline \multirow{2}{*}{ Substrato } & 5 & 20 & 35 & 50 & 65 & 80 & 95 \\
\cline { 2 - 8 } & \multicolumn{7}{c}{ Dias após o transplante (DAT) } \\
\hline S1 & $36,04 \mathrm{~b}$ & $34,79 \mathrm{~d}$ & $41,09 \mathrm{c}$ & $38,97 \mathrm{c}$ & $36,22 \mathrm{~d}$ & $38,64 \mathrm{c}$ & $38,71 \mathrm{~d}$ \\
S2 & $37,84 \mathrm{ab}$ & $39,37 \mathrm{c}$ & $43,41 \mathrm{~b}$ & $41,89 \mathrm{~b}$ & $39,54 \mathrm{c}$ & $43,44 \mathrm{~b}$ & $40,81 \mathrm{c}$ \\
S3 & $38,18 \mathrm{ab}$ & $43,41 \mathrm{~b}$ & $44,31 \mathrm{~b}$ & $42,43 \mathrm{~b}$ & $42,69 \mathrm{~b}$ & $44,47 \mathrm{ab}$ & $42,87 \mathrm{~b}$ \\
S4 & $38,88 \mathrm{a}$ & $50,04 \mathrm{a}$ & $53,72 \mathrm{a}$ & $52,59 \mathrm{a}$ & $50,04 \mathrm{a}$ & $47,15 \mathrm{a}$ & $52,54 \mathrm{a}$ \\
\hline C.V. $(\%)$ & 3,69 & 4,68 & 2,79 & 2,03 & 3,60 & 5,03 & 2,65 \\
\hline DMS & 2,25 & 3,17 & 2,06 & 1,45 & 2,45 & 3,53 & 1,87 \\
\hline
\end{tabular}

Médias seguidas pela mesma letra, na coluna, não diferem entre si, pelo teste Tukey a 5\% de probabilidade de erro. C.V. = Coeficiente de Variação. DAT: Dias Após o Transplante. S1: Solo + Areia (1:1); S2: Solo + Areia + Matéria Orgânica (1:1:1); S3: Solo + Matéria orgânica (1:1); e S4: Solo + Areia (1:1) + Osmocote $^{\circledR}$ 15-09-12 $\left(4,0 \mathrm{~g} \mathrm{~L}^{-1}\right.$ substrato).

O uso do Osmocote ${ }^{\circledR}$ e de matéria orgânica como componente dos substratos influenciou positivamente os valores de Índice SPAD, posto que, como observado nas demais variáveis, os substratos que levaram estes materiais em suas composições promoveram um melhor desenvolvimento das mudas, que consequentemente, obtiveram melhores teores de clorofila foliar e assim melhores valores de índice SPAD.

Resultados semelhantes, foram encontrados por Silva et al. (2011), que trabalhando com a produção de mudas de mangabeira (Hancornia speciosa - Apocynaceae), observaram que os melhores resultados para esta variável, foram obtidos pelos substratos que possuíam em sua composição a presença de matéria orgânica, tanto na forma de esterco quanto na forma de húmus de minhoca. Avaliando o índice SPAD do maracujazeiro-amarelo (Passiflora edulis - Passifloraceae) Silva Júnior et al. (2013) verificaram que para a adubação mineral, houve um incremento de 4,28\% no Índice SPAD para as plantas que se desenvolveram sob o uso de $100 \%$ da adubação com NK $(48,31)$ quando comparada à metade da dose $(46,24)$, indicando

Cultura Agronômica, Ilha Solteira, v.25, n.3, p.241-252, 2016 
que uma fonte de nutrientes às mudas favorece seu desenvolvimento e com isso, seu índice SPAD, apesar de não haver diferença significativa entre ambos os tratamentos.

A medida indireta da clorofila é uma técnica promissora para fornecer subsídios à recomendação de adubação nitrogenada às culturas, devido a este nutriente constituir parte de sua molécula (BUZETTI et al., 2008). De acordo com Argenta et al. (2001) é por esta razão que o teor de clorofila tem sido relacionado com o estado nutricional de diversas culturas.

Assim, com base nessas informações, pode-se dizer que o substrato S4 possui melhor estado nutricional em relação aos demais, já que promoveu maiores índices de conteúdo de clorofila, conferindo às mudas maiores as chances de alta produtividade quando adultas.

\section{ERRATA}

No artigo "DESENVOLVIMENTO INICIAL DE PLANTAS DE NONI CULTIVADAS EM DIFERENTES SUBSTRATOS”, publicado na Cultura Agronômica, Ilha Solteira, v.25, n.3, p.241-252, 2016.

Os autores relataram que, esqueceram o nome de um co-autor, assim:

Onde se lê:

"Marcela Sant'Anna Cordeiro da Silva", Antonio Flávio Arruda Ferreira², Laís Naiara Honorato Monteiro ${ }^{3}$, Aparecida Conceição Boliani ${ }^{4}$

${ }^{1}$ Mestranda em Agronomia: Horticultura - Universidade Estadual Paulista "Júlio de Mesquita Filho" - FCA, Botucatu/SP.

${ }^{2}$ Doutorando em Fitotecnia - Universidade de São Paulo - ESALQ, Piracicaba/SP.

${ }^{3}$ Mestranda em Agronomia: Sistemas de Produção - Universidade Estadual Paulista "Júlio de Mesquita Filho" - FEIS, Ilha Solteira/SP.

${ }^{4}$ Professora Dra. do Departamento de Fitotecnia, Tecnologia de Alimentos e Sócio Economia - Universidade Estadual Paulista "Júlio de Mesquita Filho" - FEIS, Ilha Solteira/SP."

Leia-se:

"Marcela Sant'Anna Cordeiro da Silva', Aparecida Conceição Boliani², Antonio Flávio Arruda Ferreira ${ }^{3}$, Laís Naiara Honorato Monteiro ${ }^{4}$, Regina Maria Monteiro de Castilho ${ }^{2}$

${ }^{1}$ Mestranda em Agronomia: Horticultura - Universidade Estadual Paulista "Júlio de Mesquita Filho" - FCA, Botucatu/SP.

${ }^{2}$ Professora Dra. do Departamento de Fitotecnia, Tecnologia de Alimentos e Sócio Economia - Universidade Estadual Paulista "Júlio de Mesquita Filho" - FEIS, Ilha Solteira/SP.

${ }^{3}$ Doutorando em Fitotecnia - Universidade de São Paulo - ESALQ, Piracicaba/SP.

${ }^{4}$ Mestranda em Agronomia: Sistemas de Produção - Universidade Estadual Paulista "Júlio de Mesquita Filho" - FEIS, Ilha Solteira/SP."

\section{CONCLUSÃO}

A partir das condições em que o experimento foi realizado, pôde-se concluir que o substrato S4, de composição solo + areia + Osmocote ${ }^{\circledR}$ 15-09-12 (1:1:1) e dosagem de 4,0 g $\mathrm{L}^{-1}$ é o mais adequado para a formação de mudas de noni.

Cultura Agronômica, Ilha Solteira, v.25, n.3, p.241-252, 2016 
A composição de substrato menos indicada para produção de mudas de noni é o substrato S1, Solo + Areia (1:1), inserindo-se assim, a alta necessidade da espécie em termos nutricionais.

\section{AGRADECIMENTOS}

Os autores agradecem ao Programa Institucional de Bolsa de Iniciação Científica (PIBIC/CNPq) pela concessão da bolsa à primeira autora.

\section{REFERÊNCIAS BIBLIOGRÁFICAS}

ARGENTA G; SILVA P. R. F; BARTOLINI C. G. Clorofila na folha como indicador do nível de nitrogênio em cereais. Ciência Rural, Santa Maria, v. 31, n. 4, p.715-722, 2001.

BACKES, M. A. Composto do lixo urbano como substrato para plantas ornamentais. 1989. 78 f. Dissertação (Mestrado em Agronomia) - Faculdade de Agronomia, Universidade Federal de Rio Grande do Sul, Porto Alegre, 1989.

BARBOSA, Z.; SOARES, I.; CRISÓSTOMO, L. A. Crescimento e absorção de nutrientes por mudas de gravioleira. Revista Brasileira de Fruticultura, Jaboticabal, v. 25, n. 3, p.519$522,2003$.

BASAR, S.; UHLENHUT, K.; HÖGGER, P.; SCHÖNE, F.; WESTENDORF, J. Analgesic and antiinflamatory activity of Morinda citrifolia L. (noni) fruit. Phytother Research Institute of Experimental and Clinical Pharmacology and Toxicology, University Clinic Hamburg, Germany. v. 24, n. 1, p.38-42, 2010.

BRASIL, E. C.; VIÉGAS, I. J. M.; SILVA, E. S. A.; GATO, R. F. Nutrição e adubação: conceitos e aplicações na formação de mudas de pimenta longa. Belém: Embrapa Amazônia Oriental, 1999. 23 p. Embrapa Amazônia Oriental. (Documentos, 13).

BUZETTI, S.; ANDREOTTI, M.; FURLANI JUNIOR, E.; VILAS-BOAS, R. L. Perspectivas de uso de métodos diagnósticos alternativos: medida indireta de clorofila. In: PRADO, R. M.; ROZANE, D. E.; VALE, D. W.; CORREIA, M. A. M.; SOUZA, H. A. (Org.). Nutrição de plantas: diagnose foliar em grandes culturas. Jaboticabal: FCAV/Capes/Fundunesp, 2008. p. 135-160.

CARNEIRO, J. G. A. Produção e controle de qualidade de mudas florestais. Curitiba: UFPR/FUPEF; Campos: UENF, 1995. 451 p.

CASAGRANDE JÚNIOR, J. G.; VOLTOLINI, J.; HOFFMANN, A.; FACHINELlO, J. Efeito de materiais orgânicos no crescimento de mudas de araçazeiro (Psidium cattleyanum Sabine). Revista Brasileira de Agrociência, Pelotas, v. 2, n. 3, p.187-191, 1996.

CUNHA, A. M.; CUNHA, G. M.; SARMENTO, R. A.; CUNHA, G. M.; AMARAL, J. F. T. do. Efeito de diferentes substratos sobre o desenvolvimento de mudas de Acacia sp. Revista Árvore, Viçosa, v. 3, n. 2, p.207-214, 2006.

Cultura Agronômica, Ilha Solteira, v.25, n.3, p.241-252, 2016 
DINALli, R. P.; CASTILHO, R. M. M.; GAZOLA, R. N. Utilização de fertilizantes de liberação lenta na produção de mudas de Vigna radiata L. Revista Científica Eletrônica de Agronomia, Garça, v. 21, n. 1, p.10-15, 2012.

EMPÓRIO DAS SEMENTES. Osmocote plus®. Disponível em: www. emporiodassementes.com.br . Acesso em: 06 jun. 2014.

EMPRESA BRASILEIRA DE PESQUISA AGROPECUÁRIA- EMBRAPA. Centro Nacional de Pesquisas de Solos. Sistema brasileiro de classificação de solos. 3.ed. Rio de Janeiro, Embrapa Solos, Brasília, Sistema de Produção de Informação, 2013. 353 p.

FERREIRA, D. F. SISVAR: a computer statistical analysis system. Ciência e Agrotecnologia (UFLA), Lavras, v. 35, n. 6, p.1039-1042, 2011.

GOMES, J. M.; PAIVA, H. N. Viveiros florestais (propagação sexuada). $3^{\mathrm{a}}$ ed. Viçosa, UFV, 2004. 116 p. (Cadernos Didáticos, 72).

GONÇALVES, J. L. M.; SANTARELLI, E. G.; MORAES NETO, S. P.; MANARA, M. P. Produção de mudas de espécies nativas: substrato, nutrição, sombreamento e fertilização. In: GONÇALVES, J. L. M.; BENEDETI, V (Eds.). Nutrição e fertilização florestal. Piracicaba, IPEF, 2000. cap. 11, p. 309-350.

HOWARD, A. S. Um testamento agrícola: expressão popular. 1. ed. São Paulo. 2007. 360 p.

KÄMPF, A. N. Substratos para floricultura: manual de floricultura. In: SIMPÓSIO BRASILEIRO DE FLORICULTURA E PLANTAS ORNAMENTAIS, 1992, Maringá. Anais... Maringá: UFPR, 1992. p. 36-43.

MARCUZZO, K. V.; MELO, B.; TEODORO, R. E. F.; ALVARENGA, C. B.; GONÇALVES, M. V.; GUIRELLI, J. E. Desenvolvimento de mudas de cafeeiro em diferentes substratos e doses de fertilizante de liberação gradual. In: SIMPÓSIO DE PESQUISA DOS CAFÉS DO BRASIL E WORKSHOP INTERNACIONAL DE CAFÉ E SAÚDE, 3. 2005, Porto Seguro, Anais... Brasília: Embrapa Café, 2005. p. 284- 285.

MENDONÇA, V.; RAMOS, J. D.; GONTIJO, T. C. A.; MARTINS, P. C. C.; DANTAS, D. J.; PIO, R.; ABREU, N. A. A. Osmocote ${ }^{\circledR}$ e substratos alternativos na produção de mudas de maracujazeiro-amarelo. Ciência e Agrotecnologia, Lavras, v. 28, n. 4, p.799-806, 2004.

MOREIRA, G. G.; REIS, F. O.; SANTOS, T. S.; LIRA, V. A.; SILVA, B. V. Acompanhamento de mudas de Morinda citrifolia cultivadas em campo submetidas a diferentes tipos de adubações. In: CONGRESSO BRASILEIRO DE AGROECOLOGIA, 8., 2013, Porto Alegre. Resumos... Porto Alegre: Cadernos de Agroecologia, 2013. 5 p. 1 CDROM.

MOREIRA, F. M. S.; MOREIRA, F. W. Características de germinação de 64 espécies de leguminosas florestais nativas da Amazônia, em condições de viveiro. Acta Amazônica, Manaus, v. 26, n. 1/2, p.3-16, 1996.

Cultura Agronômica, Ilha Solteira, v.25, n.3, p.241-252, 2016 
NASCIMENTO, L. C. S. Caracterização centesimal, composição química e atividade antioxidante do noni (Morinda citrifolia L.) cultivado no município de Zé Doca - MA. 2012. 69 f. Dissertação (Mestrado em Ciências e Tecnologia de alimentos) Universidade Federal Rural do Rio de Janeiro, Seropédica, 2012.

NEGREIROS, J. R. S.; ÁLVARES, V. S.; BRAGA, L. R.; BRUCKNER, C. H. Diferentes substratos na formação de mudas de maracujazeiro-amarelo. Revista Ceres, v. 51, n. 294, p.243-343, 2004.

OLIVEIRA, L. L. Utilização de fertilizantes de liberação lenta na produção de Coreopsis tinctoria Nutt. e Dianthus caryophyllus L. 2006. 57 f. Trabalho de Conclusão de Curso (Graduação em Agronomia) Faculdade de Engenharia, Universidade Estadual Paulista, Ilha Solteira, 2006.

SANTANA FILHO, L. G. M.; BLANK, A. F.; CARVALHO FILHO, J. L. S.; ARRIGONIBLANK, M. F.; SANTOS, M. F.; SILVA-MANN, R.; FERREIRA, R. A.; DANTAS, I. B. Avaliação do crescimento inicial de acessos de noni. In: CONGRESSO BRASILEIRO DE OLERICULTURA, 43., 2003, Recife. Resumos... Brasília: SOB, 2003. v. 21, p.1-4. 1 CDROM.

SANTOS, C. B.; LONGHI, S. J.; HOPPE, J. M.; MOSCOVICH, F. A. Efeito do volume de tubetes e tipos de substratos na qualidade de mudas de Cryptomeria japonica (L.F.) D. Don. Ciência Florestal, Santa Maria, v. 10, n. 2, p.1-15, 2000.

SILVA, E. A.; OLIVEIRA, A. C.; MENDONÇA, V.; SOARES, F. M. Substratos na produção de mudas de mangabeira em tubetes. Pesquisa Agropecuária Tropical, Goiânia, v. 41, n. 2 , p.279-285, 2011.

SILVA, L. R.; MEDEIROS, P. V. Q. de; LEITE, G. A.; SILVA, K. J. P.; MENDONÇA, V.; SILVA, G. G. Caracterização do fruto de Morinda citrifolia L. (noni). Revista Cubana de Plantas Medicinales, Ciudad de la Habana, v. 17, n. 1, p.93-100, 2012.

SILVA JÚNIOR, G. B.; CAVALCANTE, I. H. L; ALBANO, F. G.; OSAJIMA, J. A. Estado nutricional e clorofila foliar do maracujazeiro-amarelo em função de biofertilizantes, calagem e adubação com N e K. Revista de Ciências Agrárias, Lisboa, v. 36, n. 2, p.163-173, 2013.

SCHMIDT-VOGT, H.; GÜRTH, P. Eigenschaften von forstpflanzen und kultturerfolg - I. Mitteilung: Auspflanzungsversuche mit fichten-und kieferpflanzen verschiedener grossen und durchmesser. Allg Forst-u. Jagdztg, Frankfurt, v. 140, n. 6, p.132-142, 1969.

SOUSA, J. A.; SILVA NETO, P. A. F.; FERREIRA, F. V. M.; ARAÚJO, D. B.; SOUSA, J. C. R.; AQUINO, A. R. L.; SILVA, T. C.; BEZERRA, F. C. Substrato para produção de mudas de noni (Morinda citrifolia) In: CONGRESSO BRASILEIRO DE AGRONOMIA, 26., 2009, Gramado. Anais... Gramado: Confederação dos Engenheiros Agrônomos do Brasil/Sociedade de Agronomia do Rio Grande do Sul, 2009. p.1-4. 1 CD-ROM

YAMANISHI, O. K; FAGUNDES, G. R.; MACHADO FILHO, J. A.; VALONE, G. de V. Efeito de diferentes substratos e duas formas de adubação na produção de mudas de mamoeiro. Revista Brasileira de Fruticultura, Brasília, v. 26, n. 2, p.276-279, 2004. 